\title{
Hydrodynamies of a Permeable Patch in the Fluid Membrane
}

\author{
F. W. WIEGEL \\ Department of Applied Physics, Twente University of Technology, \\ P.O. Box 217, 7500 AE Enschede, The Netherlands
}

(Received 18 September 1978)

\begin{abstract}
A patch of cross-linked proteins in the fluid membrane is considered for the case in which the patch is permeable (porous) for the lipid flow in the membrane. The Bretscher flow field is studied quantitatively and the distribution of Brownian particles over the surface of the cell is given. This leads to a simple quantitative criterion for cap formation. Finally, explicit expressions for the rotational and translational diffusion coefficients of a permeable patch, as calculated from hydrodynamics, are given.
\end{abstract}

\section{Introduction}

The concept of a fluid membrane with laterally mobile lipid and protein constituents has had considerable impact on membrane biophysics. An interesting addition to this concept is duc to Bretscher (1976) who assumed that there is a continuous oriented flow of lipid molecules in the plasma membrane. This flow is due to the insertion of lipids at certain sites of the membrane and their removal through endocytosis at one specific site on the membrane. As a consequence of flow, all objects in the membrane are subject to a drag force which is directed towards one specific site. In the case of an isolated protein the Brownian movement counteracts the sweeping action of membrane flow and the resulting equilibrium situation does not differ much from a density which is constant over the whole membrane. On the other hand, in the case of a patch of cross-linked immunoglobulin molecules in the plasma membrane of a lymphocyte the diffusion constant is much smaller, and membrane flow effectively sweeps all sufficiently large patches to the vicinity of a specific site on the cell surface, i.e. capping occurs.

In this paper some hydrodynamics and statistical consequences of membrane flow are discussed. Section 2 is a derivation of various properties of the Bretscher flow field. We calculate the distribution of Brownian particles (impermeable proteins or permeable patches of cross-linked receptors) over the surface of the cell and derive a simple criterion for capping to occur. In section 3 we present explicit expressions for the 
rotational and translational diffusion coefficients of a permeable patch. Some order of magnitude estimations are the subject of the last section.

\section{Hydrodynamic Features of the Bretscher Flow Field}

Let us assume that the cell membrane is a sphere of radius $R$. It is convenient to use polar co-ordinates in such a way that the specific point at which lipids are removed from the membrane through endocytosis corresponds to the "North Pole". Let $\theta$ denote the angle which measures how far an arbitrary point of the membrane is removed from the North Pole; $0 \leqslant \theta \leqslant \pi$. New lipids are inserted in the membrane at random positions in such a way that in the stationary state a fraction $\alpha$ of the total area is renewed per unit of time. This leads to a flow field in which the velocity $v(\theta)$ is directed tangentially to the membrane, in the direction of the pole, and with magnitude

$$
v(\theta)=\alpha R(1+\cos \theta) / \sin \theta .
$$

In the stationary state the density $\rho(\theta)$ of Brownian particles (number of particles per unit area) will be the outcome of a competition between the drag force which tends to sweep the particles to the pole at $\theta=0$ and the Brownian movement which tends to spread them out uniformly over the surface. It is straightforward to show that this leads to the distribution:

$$
\rho(\theta)=\rho(\pi)[\sin (\theta / 2)]^{-2 \alpha R^{2} / D_{T}},
$$

where $D_{T}$ denotes the translational diffusion coefficient; and $\rho(\pi)$ the density at the "South Pole".

When (2) is integrated over the whole surface of the membrane we find that the integral diverges at $\theta=0$ if and only if $2 \alpha R^{2} / D_{\mathrm{T}} \geqslant 2$. This divergence is of course due to the idealized nature of the model in which all lipids are removed at one point $\theta=0$ (in reality endocytosis will occur in a small vicinity of $\theta=0$ ). In any case, the divergence of the integral of (2) over the membrane surface implies that a Brownian particle will be found at $\theta=0$ with a probability very close to unity. This gives a quantitative criterion for cap formation:

$$
\text { (cap formation) } \leftrightarrow\left(\alpha R^{2} \geqslant D_{T}\right) .
$$

In order to complete the hydrodynamic theory of cap formation we have to express $D_{T}$ in terms of the viscosity of the membrane and the dimensions of the patches. This is the subject of the next section. 


\section{Diffusion Coefficients of a Permeable Patch}

Let us represent a porous patch by a rigid cylindrical disk of radius $a$ and a height $h$ which equals the thickness of the membrane. It is assumed that the disk never protrudes out of either side of the membrane. For a particle in uniform translation with respect to the membrane the translational friction coefficient $f_{T}$ is defined as the drag force per unit relative velocity. For a particle in a state of uniform rotation the rotational friction coefficient $f_{R}$ is defined as the torque per unit angular velocity. The translational and rotational diffusion coefficients $D_{T}$ and $D_{R}$ are related to these friction coefficients by the Einstein relations:

$$
D_{T}=\frac{k_{B} T}{f_{T}} ; \quad D_{R}=\frac{k_{B} T}{f_{R}},
$$

where $k_{B}$ denotes Boltzmann's constant and $T$ the absolute temperature.

For a hard (impermeable) disk the diffusion coefficients have been calculated by Saffman \& Delbrück (1975) and by Saffman (1976). The hard disk is an appropriate model for a single globular protein, but not for a patch of cross-linked receptors in, for example, the lymphocyte membrane. These receptors are immunoglobulin molecules which are embedded in the membrane with their $F c$ fragments. When such molecules are cross-linked in a patch neighbouring $F c$ fragments are separated by distances of the order of $100 \AA$ [cf. Delisi (1976) for an introduction to antigen-antibody interactions]. Because the lipids in the membrane have cross-sections with linear dimensions of the order of $5 \AA$ these patches will be permeable to the membrane fluid.

It is, therefore, more realistic to describe a patch by a circular disk of constant permeability $K_{0}$. Operationally the permeability is defined by an experiment in which the fluid flows along an infinite plane wall made of porous matcrial. The velocity $(V)$ of the fluid in the permeable material will decrease according to the relation:

$$
V(x)=V(0) \exp \left(-x / \sqrt{K_{0}}\right)
$$

as a function of the distance $(x)$ to the wall. It turns out that stationary flow of a fluid under such circumstances can be described by the DebyeBrinkman--Bueche equation :

$$
-\vec{\nabla} P+\eta \wedge \vec{V}-\frac{\eta}{K_{0}}(\vec{V}-\vec{U})=0
$$

where $P$ denotes the pressure in the fluid; $\eta$ the viscosity; and $\vec{V}$ c.q. $\vec{U}$ the 
local velocity of the fluid, c.q. the porous material. A microscopic derivation of (6) has been given by Felderhof \& Deutsch (1975a) and a macroscopic derivation by Wiegel \& Mijnlieff (1976). Recent applications to the flow of a viscous fluid through polymer coils have been published by Ooms, Mijnlieff \& Beckers (1970); Felderhof \& Deutsch (1975a,b); Felderhof $(1975 a, b)$; Wiegel \& Mijnlieff $(1977 a, b)$; and Mijnlieff \& Wiegel (1978).

Using the Debye-Brinkman-Bueche equation we find for the rotational diffusion coefficient of a patch the expression:

$$
D_{R}=\frac{k_{B} T}{4 \pi \eta \eta h a^{2}} \frac{I_{0}(\sigma)}{I_{2}(\sigma)}
$$

The $I_{v}(\sigma)$ denote the modified Bessel functions, and

$$
\sigma=\frac{a}{\sqrt{K_{0}}}
$$

is a dimensionless quantity which measures the ratio of the radius of the patch and the penetration length $\sqrt{K_{0}}$. The derivation of (7) is given elsewhere (Wiegel, 1979a).

The calculation of the translational diffusion coefficient is more complicated because of the Stokes paradox. In order to obtain finite quantities we have to consider a porous cylinder in a sheet (thickness $h$ ) of fluid with viscosity $\eta$ which is embedded on both sides in another fluid of much lower viscosity $\eta^{\prime}$. For $\left(h \eta / a \eta^{\prime}\right) \gg 1$ we find the asymptotic result:

$$
D_{T}=\frac{k_{B} T}{4 \pi \eta h}\left\{-\gamma+\ln \left(h \eta / a \eta^{\prime}\right)+\frac{2}{\sigma^{2}}+\frac{I_{0}(\sigma)}{\sigma I_{1}(\sigma)}\right\},
$$

where $\gamma=0.5772$ denotes Euler's constant. The derivation of $(9)^{\circ}$ is given elsewhere (Wiegel, 1979b). In combination with (3) this equation gives a complete hydrodynamic theory of cap formation.

\section{Discussion}

It is clear from the results of the preceding section that the experimental determination of the rotational and translational diffusion coefficient of a patch of cross-linked immunoglobulin molecules is of considerable interest. To date, no such measurements have been reported in the literature.

Some order of magnitude estimations of related quantities are as follows: The viscosity $\eta^{\prime}$ is of the order of the viscosity of water: $\eta^{\prime} \cong 0.01 \mathrm{~g} \mathrm{~cm}^{-1} \mathrm{~s}^{-1}$ at $25^{\circ} \mathrm{C}$. The lipid bilayer has a thickness $h \cong 40 \AA$ and a viscosity $\eta \cong 2$ $\mathrm{g} \mathrm{cm}^{-1} \mathrm{~s}^{-1}$ at $25^{\circ} \mathrm{C}$ (Prives \& Shinitzky, 1977). The translational diffusion 
coefficient of the lipids themselves has been estimated as $D_{T} \cong 2 \times 10^{-7}$ $\mathrm{cm}^{2} \mathrm{~s}^{-1}$ at $25^{\circ} \mathrm{C}$ (Fahey et al., 1977). Smaller diffusion coefficients have been reported for rhodopsin in the frog photoreceptor membrane: $D_{T} \cong 3.5 \times 10^{-9} \mathrm{~cm}^{2} \mathrm{~s}^{-1} ; D_{R} \cong 5 \times 10^{4} \mathrm{~s}^{-1}$ at $20^{\circ} \mathrm{C}$ (Poo \& Cone, 1974; Cone, 1972). This protein has a radius $a \cong 20 \AA$. Even smaller diffusion coefficients were found for integral proteins in the human erythrocyte membrane: $D_{T} \cong 3 \times 10^{-11} \mathrm{~cm}^{2} \mathrm{~s}^{-1}$ at $30^{\circ} \mathrm{C}$ (Fowler \& Branton, 1977) and $D_{R} \cong 10^{3} \mathrm{~s}^{-1}$ at $22^{\circ} \mathrm{C}$ (Cherry et al., 1976). These proteins have a radius $a \cong 40 \AA$.

The average velocity $\langle v\rangle$ of the flow field over the surface of the cell is calculated from $(1):\langle v\rangle=\frac{1}{2} \pi \alpha R$. For a lymphocyte with $R \cong 5 \times 10^{-4} \mathrm{~cm}$, Bretscher (1976) estimates $\langle v\rangle \cong 5 \times 10^{-6} \mathrm{~cm} \mathrm{~s}^{-1}$. Substituting into (3) one expects permeable patches to form a cap if $D_{r} \lesssim 1.6 \times 10^{-9} \mathrm{~cm}^{2} \mathrm{~s}^{-1}$.

The author is indebted to Dr W. B. Goad and Dr G. I. Bell of the Los Alamos Scientific Laboratory and to Dr A. S. Perelson of Brown University for discussions and a correspondence.

\section{REFERENCES}

BRETSCHER, M. S. (1976). Nature 260, 21.

Cherry, R. J., Bürkli, A., Busslinger, M., SChneider, G. \& Parish, G. R. (1976). Nulure 263 , 389.

Cone, R. A. (1972). Nature new Biol. 236, 39.

DeLisI, C. (1976). Antigen-Antibody Interactions. New York: Springer.

Fahey, P. F., Koppel, D. E., BaraK, L. S., WOlf, D. E., El.sON, E. L. \& WebB, W. W. (1977), Science 195, 305.

FelderhoF, B. U. \& Deutsch, J. M. (1975a). J. chem. Phys. 62, 2391.

Felderhor, B. U. \& Deutsch, J. M. (1975b). J. chem. Phys. 62, 2398.

FELDERHOF, B. U. (1975a). Physica 80A, 63.

FeLDERHOF, B. U. (1975b). Physica 80A, 172.

Fowler, V. \& BRANTON, D. (1977). Nature 268, 23.

Mundieff, P. F. \& Wiegel, F. W. (1978). J. Pol. Sc.: Physics Ed. 16, 245.

Ooms, G., MunliefF, P. F. \& Beckers, H. L. (1970). J. chem. Phys. 53, 4123.

Poo, M. \& Cone, R. A. (1974). Nature 247, 438.

PRIVES, J. \& SHINITZKY, M. (1977). Nature 268, 761.

SAfFMAN, P. G. (1976). J. Fluid Mech. 73, 593.

SaFfMan, P. G. \& DelBrÜCK, M. (1975). Proc. natn. Acad. Sci. U.S.A. 72, 3111.

Wiegel, F. W. \& MiJnlieff, P. F. (1976). Physica 85A, 207.

Wiegel, F. W. \& Minlieff, P. F. (1977a). Polymer 18, 636.

WIEGEL, F. W. \& MusNLIEFF, P. F. (1977b). Physica 89A, 385.

WIEGEL, F. W. (1979a). Rotational friction coefficient of a permeable cylinder in a viscous fluid. To appear in Phys. Letts A.

WIEGEL, F. W. (1979b). Translational friction coefficient of a permeable cylinder in a sheet of viscous fuid. To appear. 